\title{
Determination of The Harvesting Time of Hass Cultivar in Antalya Conditions
}

\author{
Süleyman BAYRAM ${ }^{1} \mathbb{D}$, Seyla TEPE $^{1}$
}

1Batı Akdeniz Agricultural Research Institute, 07100, Antalya / Turkey

\author{
Article History \\ Received 26 July 2019 \\ Accepted 07 September 2020 \\ First Online 01 October 2020
}

\section{Corresponding Author \\ E-mail: slymnbayram@gmail.com}

\author{
Keywords \\ Avocado \\ Hass \\ Maturity \\ Ripening \\ Harvest \\ Harvest period
}

\begin{abstract}
Avocado cultivation in the world and Turkey, especially Hass cultivar, increases rapidly in recent years. The fruit quality of avocado is directly related to harvest maturity and post-harvest ripening process and the identification of maturity index has a very large commercial importance. For this purpose; in the fruit samples of Hass cultivar taken at 15-20 days interval from October to June, some fruit quality traits (dry matter, flesh firmness, weight loss, taste, colour of fruit skin and flesh) during the harvest and ripening process and relationships between of these parameters were analysed. As a result, it was found that the most reliable maturity index was the dry matter (DM) content and that there was a direct relationship between its accumulation and the harvesting time. In cases these index values were insufficient, the other postharvest analyses (taste, fruit skin colour, fruit hardness and weight loss) made a contribution to determination of maturity. During the ripening process, very high levels of a positive correlation between harvest time and dry matter ( $r=0.92$ to 0.96 ) was observed. According to the fruit maturity of the Hass cultivar, three different harvest periods were defined as early, optimum (most suitable) and late harvests. It was determined that from early October to late December as early harvest (23-25\% DM), from January to late May as optimum harvest (26-37\% DM), and from beginning to end of June as late harvest ( $\geq 38 \% \mathrm{DM})$.
\end{abstract}

\section{Introduction}

Recognizability of avocado fruit gradually increases and is much more involved in the healthrelated social networks in recent years. There is very high demand in the World for the production and consumption of avocado due to the positive effects on human health as a functional food and the high nutritional value (Anonymous, 2006). As a result, the consumption habits of avocado fruit develop, production areas increase, and fruits find consumers at high prices in the market. Avocado is cultivated in approximately 50 countries on 5 continents in the World as a subtropical fruit species
(Zentmyer, 1987; Knight, 2002). There is two major avocado market in the world that are the United States and European Union countries (Naamani, 2007). Moreover, these two major markets comprise more than $90 \%$ of total imports in the World (Naamani, 2007; FAO, 2019). Japan and Canada follow these two major markets as smaller markets (FAO, 2019). In the world avocado market, a large portion of imports and exports consist of Hass cultivar (Naamani, 2007; Hernández et al., 2015) and it is the main cultivar of the avocado market with its superior fruit quality (Anonymous, 2005; Naamani, 2007). Furthermore, Hass is a very important cultivar in terms of post-harvest transport, 
storage suitability, and high yield (Newett et al., 2002; Naamani, 2007). Besides, especially under cool subtropical cultivation conditions, after reaching physiological maturity, it has 'storage on the tree' characteristic that is an advantage for the market (Whiley et al., 1996; Hofman et al., 2002).

For the climacteric fruits such as avocado, in an export chain where supplying to reach distant markets as high quality of fruits, harvesting of the fruits in the right time with regard to the grade of maturity is a very important procedure (Ginsberg, 1985), and there may be significant differences in the composition of the fruit according to the harvesting time (Gonzales et al., 1992; Whiley et al., 1992; Ozdemir et al., 2003, Ozdemir and Topuz, 2004; Villa-Rodríguez et al., 2010). Harvesting of fruits in the early or late period to benefit from the high price advantage in the market cause of some great problems in ripening and quality of fruits. While irregular ripening, wrinkling, hardening and rotting of fruit flesh are observed in early harvest, deteriorating in fruit flesh, cracking and abscission in fruits are seen in late harvest (Young and Lee, 1978; Lee et al., 1983; Flitsanov et al., 2000; Hofman et al., 2002; Kassim et al., 2013; Carvalho et al., 2014; Magzawa and Tesfay, 2015). Furthermore, long flowering period, a low percentage of the fruit set, and not to ripening on the tree causes a heterogeneous and unpredictable fruit structure in the post-harvest period of Hass cultivar (Hernández et al., 2015). Therefore; during transport and storage of the avocado, harvesting time has a very important role to play on the shelf life and ripening (Osuna-Garcia et al., 2010; OsunaGarcia et al., 2011; Carvalho et al., 2014), and determination of the most appropriate harvest period for high-value marketing of fruit may be the most critical decision need to be given.

Maturity dates of avocado fruits may vary widely even in a certain region and short distances (Coggins, 1984). The basis of the determination of the harvesting time in fruit is comprised of the maturity (Mizrach et al., 1999; Arpaia et al., 2003; Wedding et al., 2011) and it has a great importance for the start of the ripening after the harvest (Vakis et al., 1985; Woolf et al., 2003). The maturity of the fruit is most likely affected by many cultural and environmental factors along with altitude, location and direction (slope) of the garden (Coggins, 1984). Furthermore, for the determination of internal (fruit flesh texture and flavour) and external (visual appearance) eating qualities of mature avocado fruits, the maturity level of the fruit in harvest is the most important factor (Vakis et al., 1985; Magzawa and Tesfay, 2015). However, as in many biological subjects, in some cases, the definition of maturity can be quite complex. The external appearance of avocado fruit, as is known in many fruit species found in the horticulture, cannot adequately define the maturity (Osuna-Garcia et al, 2010; Wedding et al., 2011), and the internal structure of the fruit or the quality of eating cannot generally be an accurate guide alone for determining maturity (Lee et al., 1983; Wedding et al., 2011). Avocado should be harvested according to the maturity defined as physiological and horticultural characteristics (Magzawa and Tesfay, 2015).

A reliable maturity index is necessary for determining the harvesting time depending on fruit development of avocado and a measurable parameter should change according to the harvest (Woolf et al., 2003). Although some quality characteristics in fruit need to be defined for acceptable taste, there may also be some difficulties in the determination of these standards (Young and Lee, 1978; Lee, 1981a; Woolf et al., 2003). However, for the determination of the maturity of avocado, the dry matter content of the fruit flesh is still the most reliable index (Mizrach et al., 1999) and the other important standard is the taste (Lee, 1981; Mizrach et al., 1999; Kassim et al., 2013). Therefore; in many countries that produce avocados, the dry matter content of the fruit flesh is used as the optimal maturity standard to prevent the marketing of low-quality and immaturity fruit (Hofman et al., 2002; Woolf et al., 2003; Kassim et al., 2013; Carvalho et al., 2014). As the dry matter content of the fruit flesh increases, the acceptability of the fruit is positively affected (Arpaia et al., 2003).

In this study, it is aimed to determine of the fruit maturity standards and harvesting time of Hass cultivar in Antalya condition. In the fruit samples taken at certain periods, the changes of some fruit quality criteria were observed during the postharvest maturation process (at ambient temperature in the laboratory). Analyses were carried out on the beginning day, $7^{\text {th }}$ and $14^{\text {th }}$ days of the post-harvest ripening period. As a result of the study, according to the maturity and ripening of the fruit, the harvest period of Hass cultivar was separated to three harvest interval as early, optimum and late.

\section{Material and Method}

\subsection{Material}

This research was carried out at the Fruit Growing Department of Batı Akdeniz Agricultural Research Institute in Antalya between 2010 and 2013. The 20-year old trees of Hass cultivar were used as the plant material of the study.

\subsection{Method}

The harvesting period of the first year were done from October-2010 to June-2011 and the second year studies were conducted between October 2012 and June 2013. Due to frost damage and periodicity, the experiment cannot be carried out at the harvest periods in 2011-2012. Twelve fruit 
samples were taken from the four sides of trees for each replication at $15-20$ days intervals during the harvest period. The harvested fruits were immediately transported to the laboratory and the first analyses were done on the same day. During the harvest period between October-June, the ripening process of fruits was carried out at the room temperature in the laboratory as in the study of Ozdemir and Topuz (2004), and the samples were kept for 7 and 14 days at this condition. Additionally, it was observed that the average temperature in the laboratory condition varied between $18^{\circ} \mathrm{C}-30^{\circ} \mathrm{C}$, while the relative humidity ranged between $25 \%-85 \%$.

According to Lee and Coggins (1982) dry weight (\%), fruit flesh firmness (N) with $3 \mathrm{~mm}$ tip and T.R. Turoni 53200 (FT-327) penetrometer, and fruit weight loss (\%) were measured. Furthermore, according to C.I.E. $L$ * $a{ }^{*} b$ * colour system belonging to Zerbini and Polesello (1984), the colour of the fruit skin and of the fruit flesh were determined with Minolta CR-400 chromameter. Additionally, the Chroma $\left(\mathrm{C}^{*}\right)$ and hue (h0) values were calculated as reported by McGuire (1992). Taste analyses were evaluated according to their colour, texture and flavour. The taste evaluations were determined with a score of at least 5 panellists according to IPGRI's 1-5 (1: Very poor, 2: Poor, 3: Medium, 4: Good, 5: Very good) scoring principle. Statistical analysis, the physical and chemical traits of the Hass cultivar samples that were taken at different harvest times were analysed using the JUMP software program and differences between means were determined by LSD test. The experiment was carried out in a completely randomized design (CRD) with three replications and two trees at each replication.

\section{Results and Discussion}

During the harvest and harvesting process; dry matter, flesh firmness, weight loss and taste values are given in Table 1, while fruit skin colour and flesh colour (Lab) values are given in Tables 2 and 3, respectively. According to the seasonal distribution of each analysis made at harvest ( $0^{\text {th }}$ day) and in the ripening process $\left(7^{\text {th }}\right.$ and $14^{\text {th }}$ days) in both harvest periods (Table 1); although in the dry weight content (\%) increased in between the months of October and June, there was no correlation between the analyses $\left(0^{\text {th }}, 7^{\text {th }}\right.$ and $14^{\text {th }}$ days $)$ made for each harvest.

The fruit quality and market value of avocado are directly affected by the maturity level of the harvested fruit (Olarewaju, 2014) and as the maturity level of the fruit changes during the harvest period, the optimum harvesting time of fruit need to be determined (Olarewaju, 2014; Bayram and Tepe, 2018). Dry matter values of the fruit in avocado are the most important criteria for the determination of harvest maturity (Mizrach et al., 1999; Kassim et al., 2013; Calvalho et al., 2014). In the early and late harvest of avocado, an uneven ripening process occurs in fruits (Hofman et al., 2000; Osuna-Garcia et al., 2011; Kassim et al., 2013). It is a well-known fact that the dry weight values of the fruit increase during the harvest period and therefore the fruit quality changes in a positive aspect (Arpaia et al., 2003; Ozdemir et al., 2003; Parodi et al., 2007; Osuna-Garcia et al., 2011). In case the Hass cultivar were left on the tree during the winter months in Israel, the fruits continued to the physical development and fruit weight increased until the average of 31-43 g per unit (Winer et al., 2007). However, in New Zealand between September and April, it was stated that the average dry matter increased from $24.6 \%$ to $36.4 \%$ in Te Puke and from $24.1 \%$ to $32.3 \%$ in Far North cultivar (Requejo-Tapia et al., 1999). In addition, during the import period of Hass in New Zealand (between 25 months according to regions), the daily increase in dry matter percentage was reported to be in a linear structure between $0.06 \%$ and $0.11 \%$ (Pak et al., 2003). In studies that were done with Hass cultivar (Ozdemir and Topuz, 2004; Bayram and Aşkın, 2006; Osuna-Garcia et al., 2011) were observed that the dry matter content increased during the harvest period according to the degree of ripening. Additionally, in other studies that have been done in Turkey (Ozdemir and Topuz, 2004; Bayram and Aşkın, 2006) and in Mexico (OsunaGarcia et al., 2011), it was observed that the dry matter content of Hass cultivar increased depending on maturation level of fruit during the harvest period. Similarly, the dry matter content in this study increased to a certain level during both harvest periods (from October to June) and it was found to be the most important maturity indicator. However, in the ripening process of fruits $\left(1^{\text {st }}, 4^{\text {th }}\right.$, and $8^{\text {th }}$ days), although there are significant differences in dry matter and oil content values, it has been reported that there have been very few changes when compared to the fruits remaining on the tree (Ozdemir and Topuz, 2004). In another study conducted in Israel, it was determined that dry matter content of avocado did not change after harvest (Degani et al., 1986). In a way to support previously reported results, it was found that dry matter content was not a reliable index in determining the physiological changes associated with the postharvest ripening process in this study.

In the flesh firmness values $(\mathrm{N})$, in the beginning analyses (0th day) during both harvest periods, although there was no regular a relationship in the early stages of the harvesting time, gradually decreasing was detected in the progressive process. The process of ripening in the postharvest was generally completed between 7 and 14 days. In this ripening process, with the softening of the fruit flesh, the firmness decreased up to zero level. Flesh 
Table 1. The values of dry matter content (\%), fruit flesh firmness $(\mathrm{N})$, weight loss (\%) and taste (1-5) during harvest and post-harvest ripening process of Hass cultivar (2010-2011 and 2012-2013 harvest period)

\begin{tabular}{|c|c|c|c|c|c|c|c|c|c|c|c|c|c|c|}
\hline \multirow{3}{*}{$\begin{array}{l}\text { Harvesting time } \\
05 \text { October } 2010\end{array}$} & \multicolumn{6}{|c|}{ Dry matter $(\%)^{*}$} & \multirow{3}{*}{$\frac{\text { LSD }^{* *}}{1.07}$} & \multicolumn{6}{|c|}{ Flesh firmness $(\mathrm{N})^{*}$} & \multirow{3}{*}{$\begin{array}{r}\text { LSD }^{* *} \\
8.11\end{array}$} \\
\hline & \multicolumn{2}{|c|}{$0^{\text {th }}$ day } & \multicolumn{2}{|c|}{$7^{\text {th }}$ day } & \multicolumn{2}{|c|}{$14^{\text {th }}$ day } & & \multicolumn{2}{|c|}{$0^{\text {th }}$ day } & \multicolumn{2}{|c|}{$7^{\text {th }}$ day } & \multicolumn{2}{|c|}{$14^{\text {th }}$ day } & \\
\hline & 19.77 & $\mathrm{Ah}$ & 20.68 & $\mathrm{Ah}$ & 20.63 & $\mathrm{Ag}$ & & 53.14 & d & 40.04 & $\mathrm{~g}$ & 8.34 & $\mathrm{~d}$ & \\
\hline 19 October & 19.60 & Ah & 20.63 & Ah & 20.93 & $\mathrm{Ag}$ & 1.92 & 59.93 & bc & 52.19 & bd & 20.16 & b & 33.26 \\
\hline 03 November & 21.57 & Agh & 20.49 & Ah & 21.03 & $\mathrm{Ag}$ & 2.02 & 61.51 & $a b c$ & 54.75 & $a b$ & 0.00 & $\mathrm{e}$ & 3.83 \\
\hline 23 November & 23.12 & Afg & 22.63 & Agh & 22.83 & Afg & 1.19 & 62.92 & a & 54.21 & $\mathrm{ac}$ & 0.00 & e & 7.11 \\
\hline 12 December & 24.37 & Aef & 23.62 & Agh & 23.51 & Aeg & 4.23 & 62.32 & $a b$ & 57.86 & a & 0.00 & e & 4.12 \\
\hline 29 December & 24.43 & Aef & 25.04 & $\mathrm{Ag}$ & 26.52 & $\mathrm{Ae}$ & 4.01 & 62.38 & $a b$ & 54.86 & $a b$ & 38.41 & a & 12.01 \\
\hline 13 January 2011 & 26.20 & Ade & 25.99 & Afg & 25.29 & Aef & 3.56 & 59.22 & c & 43.86 & eg & 8.88 & $\mathrm{~cd}$ & 29.88 \\
\hline 17 February & 27.77 & $\mathrm{Ad}$ & 28.68 & Aef & 26.53 & $\mathrm{Ae}$ & 4.27 & 54.86 & d & 1.53 & 1 & 0.00 & $\mathrm{e}$ & 7.29 \\
\hline 10 March & 31.84 & $A b c$ & 29.65 & Ade & 30.49 & $\mathrm{Ad}$ & 2.93 & 59.65 & $\mathrm{bc}$ & 45.49 & eg & 0.00 & $\mathrm{e}$ & 7.49 \\
\hline 23 March & 30.68 & $A c$ & 30.90 & Ace & 31.17 & Acd & 3.95 & 55.51 & d & 10.95 & $\mathrm{~h}$ & 0.00 & e & 31.70 \\
\hline 08 April & 30.94 & $A b c$ & 32.78 & Acd & 34.39 & $A b c$ & 3.56 & 52.62 & de & 43.75 & eg & 14.44 & bc & 31.84 \\
\hline 25 April & 33.39 & $A b$ & 34.19 & $A b c$ & 33.93 & Abd & 4.27 & 53.93 & d & 47.83 & df & 8.50 & $\mathrm{~cd}$ & 15.69 \\
\hline 10 May & 33.17 & $A b c$ & 33.63 & $A b c$ & 35.21 & Aab & 5.13 & 53.66 & d & 48.65 & $\mathrm{ce}$ & 0.00 & $\mathrm{e}$ & 1.43 \\
\hline 24 May & 37.50 & $\mathrm{Aa}$ & 36.67 & $A b$ & 37.08 & Aab & 7.57 & 54.21 & $d$ & 46.85 & df & & $2 F^{* * * *}$ & 5.70 \\
\hline 13 June & 36.45 & $\mathrm{Ba}$ & 40.29 & $\mathrm{Aa}$ & 38.37 & $\mathrm{ABa}$ & 3.18 & 49.74 & e & 42.50 & $\mathrm{fg}$ & & $\mathrm{RF}^{* * * *}$ & 4.49 \\
\hline $\mathrm{LSD}^{* * *}$ & 2.62 & & 3.43 & & 3.50 & & & 2.89 & & 5.56 & & 5.97 & & \\
\hline 08 October 2012 & 19.23 & $\mathrm{Bf}$ & 20.69 & $\mathrm{ABe}$ & 25.06 & $\mathrm{Ag}$ & 5.61 & 55.08 & $\mathrm{~b}$ & 45.85 & $a b$ & 30.40 & $\mathrm{a}$ & 26.06 \\
\hline 05 November & 22.48 & Aef & 22.80 & $\mathrm{Ae}$ & 25.15 & Afg & 3.18 & 65.70 & a & 55.08 & a & 5.80 & b & 25.59 \\
\hline 21 November & 23.14 & Aef & 24.39 & Ade & 24.22 & $\mathrm{Ag}$ & 4.71 & 52.38 & $\mathrm{bc}$ & 37.35 & bc & 5.64 & b & 38.78 \\
\hline 12 December & 24.94 & ABde & 27.76 & Acd & 24.35 & $\mathrm{Ag}$ & 2.92 & 49.44 & $\mathrm{~cd}$ & 25.41 & def & 0.00 & c & 37.69 \\
\hline 03 January 2013 & 25.78 & Ace & 28.66 & Acd & 27.31 & Aeg & 5.08 & 50.58 & c & 32.93 & $\mathrm{~cd}$ & 0.00 & c & 27.18 \\
\hline 24 January & 28.38 & Acd & 28.91 & Acd & 29.60 & Adf & 4.80 & 45.36 & e & 27.70 & de & 0.00 & c & 36.50 \\
\hline 12 February & 30.20 & $\mathrm{Bbc}$ & 32.09 & $A b c$ & 31.53 & ABde & 1.54 & 45.76 & e & 4.25 & $\mathrm{~g}$ & 0.00 & c & 16.23 \\
\hline 06 March & 33.90 & Aab & 32.16 & $A b c$ & 33.16 & Acd & 3.42 & 44.87 & ef & 0.00 & $\mathrm{~g}$ & 0.00 & c & 2.14 \\
\hline 28 March & 34.35 & $\mathrm{Bab}$ & 35.39 & $\mathrm{Bab}$ & 41.12 & Aab & 2.81 & 44.13 & eg & 0.00 & g & 0.00 & c & 4.90 \\
\hline 17 April & 34.21 & Aab & 35.31 & Aab & 37.52 & $A b c$ & 12.05 & 46.34 & de & 0.00 & g & 0.00 & c & 2.75 \\
\hline 14 May & 38.15 & $\mathrm{Aa}$ & 38.26 & $\mathrm{Aa}$ & 38.44 & $A b$ & 11.92 & 40.94 & g & 20.10 & ef & 0.00 & c & 3.98 \\
\hline 04 June & 38.28 & $\mathrm{Aa}$ & 37.82 & $\mathrm{Aa}$ & 44.03 & $\mathrm{Aa}$ & 8.62 & 42.09 & $\mathrm{fg}$ & 17.16 & $\mathrm{f}$ & 0.00 & c & 7.66 \\
\hline LSD $^{* * *}$ & 4.67 & & 4.59 & & 4.46 & & & 3.22 & & 9.55 & & 4.96 & & \\
\hline Harvesting Time & & & Weight I & oss $(\%$ & & & SD & & & Taste (1 & $-5)^{*}$ & & & \\
\hline marvestung ime & & day & & day & $14^{\text {th }}$ & day & S & & day & $7^{\text {th }} d$ & & $14^{\text {th }} \mathrm{C}$ & day & LSD \\
\hline 05 October 2010 & 0.00 & C & 13.32 & $\mathrm{Ba}$ & 20.44 & $\mathrm{Aa}$ & 1.32 & 0.00 & A & 0.00 & $\mathrm{Ad}$ & 0.83 & $A b$ & 0.88 \\
\hline 19 October & 0.00 & $\mathrm{C}$ & 8.82 & $\mathrm{Bc}$ & 15.11 & Ac & 1.49 & 0.00 & B & 0.00 & $\mathrm{Bd}$ & 4.00 & $\mathrm{Aa}$ & 1.00 \\
\hline 03 November & 0.00 & C & 5.87 & $\mathrm{Bd}$ & 12.33 & $\mathrm{Ad}$ & 0.36 & 0.00 & B & 0.00 & $\mathrm{Bd}$ & 4.33 & $\mathrm{Aa}$ & 0.88 \\
\hline 23 November & 0.00 & C & 5.42 & $\mathrm{Bd}$ & 13.09 & $\mathrm{Ad}$ & 1.10 & 0.00 & B & 0.00 & $\mathrm{Bd}$ & 4.50 & $\mathrm{Aa}$ & 0.58 \\
\hline 12 December & 0.00 & C & 4.10 & Bef & 8.87 & Aeg & 1.80 & 0.00 & B & 0.00 & $\mathrm{Bd}$ & 4.33 & $\mathrm{Aa}$ & 0.67 \\
\hline 29 December & 0.00 & C & 4.38 & $\mathrm{Be}$ & 8.67 & Afg & 0.71 & 0.00 & A & 0.00 & $\mathrm{Ad}$ & 0.00 & $A b$ & 0.00 \\
\hline 13 January 2011 & 0.00 & C & 4.11 & Bef & 8.70 & Afg & 0.46 & 0.00 & B & 3.50 & Ac & 4.17 & $\mathrm{Aa}$ & 1.05 \\
\hline 17 February & 0.00 & C & 5.39 & $\mathrm{Bd}$ & 8.25 & $\mathrm{Ag}$ & 0.75 & 0.00 & C & 4.00 & $\mathrm{Bb}$ & 4.67 & $\mathrm{Aa}$ & 0.67 \\
\hline 10 March & 0.00 & C & 3.48 & $\mathrm{Bf}$ & 9.69 & Aeg & 0.52 & 0.00 & B & 0.00 & $\mathrm{Bd}$ & 4.67 & $\mathrm{Aa}$ & 0.67 \\
\hline 23 March & 0.00 & C & 6.03 & $\mathrm{Bd}$ & 10.27 & Aef & 0.86 & 0.00 & B & 4.83 & $\mathrm{Aa}$ & 4.67 & $\mathrm{Aa}$ & 0.75 \\
\hline 08 April & 0.00 & C & 6.05 & $\mathrm{Bd}$ & 10.46 & $\mathrm{Ae}$ & 1.26 & 0.00 & B & 0.00 & $\mathrm{Bd}$ & 4.67 & $\mathrm{Aa}$ & 0.33 \\
\hline 25 April & 0.00 & C & 5.88 & $\mathrm{Bd}$ & 12.66 & $\mathrm{Ad}$ & 1.29 & 0.00 & B & 0.00 & $\mathrm{Bd}$ & 4.50 & $\mathrm{Aa}$ & 0.58 \\
\hline 10 May & 0.00 & $\mathrm{C}$ & 4.44 & $\mathrm{Be}$ & 10.58 & $\mathrm{Ae}$ & 0.82 & 0.00 & B & 0.00 & $\mathrm{Bd}$ & 4.17 & $\mathrm{Aa}$ & 0.33 \\
\hline 24 May & 0.00 & C & 8.29 & $\mathrm{Bc}$ & 18.61 & $A b$ & 3.62 & 0.00 & A & 0.00 & $\mathrm{Ad}$ & & $F^{\star * \star \star *}$ & 0.00 \\
\hline 13 June & 0.00 & $\mathrm{C}$ & 10.41 & $\mathrm{Bb}$ & 21.85 & $\mathrm{Aa}$ & 2.19 & 0.00 & A & 0.00 & $\mathrm{Ad}$ & & $\mathrm{F}^{\star \star \star \star \star *}$ & 0.00 \\
\hline LSD $^{* * *}$ & 0.00 & & 0.74 & & 1.71 & & & 0.00 & & 0.24 & & 0.91 & & \\
\hline 08 October 2012 & 0.00 & C & 13.18 & $\mathrm{Ba}$ & 29.87 & $\mathrm{Aa}$ & 2.80 & 0.00 & A & 0.00 & Ad & 0.00 & Af & 0.00 \\
\hline 05 November & 0.00 & $\mathrm{C}$ & 8.44 & $\mathrm{Bc}$ & 18.42 & Acd & 4.16 & 0.00 & B & 0.00 & $\mathrm{Bd}$ & 2.50 & $\mathrm{Ae}$ & 0.58 \\
\hline 21 November & 0.00 & C & 7.06 & $\mathrm{Bd}$ & 16.05 & $\mathrm{Ad}$ & 2.78 & 0.00 & B & 0.00 & $\mathrm{Bd}$ & 2.33 & $\mathrm{Ae}$ & 0.88 \\
\hline 12 December & 0.00 & C & 6.93 & $\mathrm{Bd}$ & 10.85 & Aeg & 1.75 & 0.00 & B & 3.00 & $A c$ & 2.33 & $\mathrm{Ae}$ & 1.05 \\
\hline 03 January 2013 & 0.00 & C & 5.19 & $\mathrm{Be}$ & 7.87 & $\mathrm{Ag}$ & 1.88 & 0.00 & B & 0.00 & $\mathrm{Bd}$ & 4.17 & $A b c$ & 0.67 \\
\hline 24 January & 0.00 & C & 6.22 & Bde & 9.47 & Afg & 1.03 & 0.00 & C & 3.33 & $\mathrm{Bc}$ & 4.33 & $A b$ & 0.94 \\
\hline 12 February & 0.00 & C & 5.53 & $\mathrm{Be}$ & 9.91 & Afg & 1.44 & 0.00 & C & 5.00 & $\mathrm{Aa}$ & 4.67 & $\mathrm{Bab}$ & 0.33 \\
\hline 06 March & 0.00 & C & 5.22 & $\mathrm{Be}$ & 9.57 & Afg & 1.47 & 0.00 & C & 4.67 & $\mathrm{Ba}$ & 5.00 & $\mathrm{Aa}$ & 0.33 \\
\hline 28 March & 0.00 & C & 5.99 & Bde & 14.18 & Adf & 4.94 & 0.00 & C & 4.50 & $\mathrm{Aa}$ & 3.50 & $\mathrm{Bd}$ & 0.00 \\
\hline 17 April & 0.00 & C & 6.18 & Bde & 15.68 & Ade & 0.94 & 0.00 & C & 5.00 & $\mathrm{Aa}$ & 3.67 & Bcd & 0.33 \\
\hline 14 May & 0.00 & C & 9.68 & $\mathrm{Bb}$ & 23.78 & $A b$ & 12.97 & 0.00 & B & 3.67 & $A b$ & 0.00 & $\mathrm{Bf}$ & 0.33 \\
\hline 04 June & 0.00 & $\mathrm{C}$ & 10.77 & $\mathrm{Bb}$ & 22.18 & $A b c$ & 4.03 & 0.00 & B & 0.00 & B & 2.17 & $\mathrm{Ae}$ & 0.33 \\
\hline LSD $^{* * *}$ & 0.00 & & 1.22 & & 5.10 & & & 0.00 & & 0.60 & & 0.61 & & \\
\hline
\end{tabular}


Table 2. The values of fruit skin colour $\left(L^{*} C{ }^{\star} h^{\circ}\right)$ during harvest and post-harvest ripening process of Hass cultivar (201011 and 2012-13 harvest period)

\begin{tabular}{|c|c|c|c|c|c|c|c|c|c|c|c|c|c|c|c|c|c|c|}
\hline \multirow{3}{*}{$\begin{array}{c}\text { Harvesting time } \\
05 \text { October } 2010\end{array}$} & \multicolumn{6}{|c|}{$L\left({ }^{*}\right)$} & \multicolumn{6}{|c|}{$C\left(^{*}\right)$} & \multicolumn{6}{|c|}{$h^{\circ}\left(^{*}\right)$} \\
\hline & \multicolumn{2}{|c|}{$0^{\text {th }}$ day } & \multicolumn{2}{|c|}{$7^{\text {th }}$ day } & \multicolumn{2}{|c|}{$14^{\text {th }}$ day } & \multicolumn{2}{|c|}{$0^{\text {th }}$ day } & \multicolumn{2}{|c|}{$7^{\text {th }}$ day } & \multicolumn{2}{|c|}{$14^{\text {th }}$ day } & \multicolumn{2}{|c|}{$0^{\text {th }}$ day } & \multicolumn{2}{|c|}{$7^{\text {th }}$ day } & \multicolumn{2}{|c|}{$14^{\text {th }}$ day } \\
\hline & 34.16 & $\mathrm{bc}$ & 33.65 & $\mathrm{ac}$ & 31.34 & bc & 19.53 & ce & 18.78 & ad & 12.66 & $\mathrm{bc}$ & 56.96 & $a b$ & 55.22 & $a$ & 66.09 & a \\
\hline 19 October & 33.50 & $\mathrm{bd}$ & 32.43 & $\mathrm{bd}$ & 32.45 & $\mathrm{~b}$ & 19.60 & ce & 16.92 & ce & 16.97 & $a b$ & 55.38 & $a b$ & 56.23 & a & 58.17 & a \\
\hline 03 November & 35.99 & $a$ & 35.02 & a & 35.51 & $a$ & 21.47 & ae & 18.18 & ad & 19.82 & a & 51.08 & $a b$ & 55.06 & a & 52.90 & a \\
\hline 23 November & 33.44 & $\mathrm{bd}$ & 34.08 & $\mathrm{ac}$ & 28.60 & ce & 17.28 & e & 16.62 & df & 6.87 & $d f$ & 55.03 & $a b$ & 56.05 & a & -53.66 & C \\
\hline 12 December & 32.71 & $\mathrm{~cd}$ & 34.10 & $\mathrm{ac}$ & 32.74 & $a b$ & 20.66 & be & 19.20 & ad & 13.67 & bc & 55.22 & $a b$ & 54.10 & a & 70.44 & a \\
\hline 29 December & 33.58 & $\mathrm{bd}$ & 33.53 & $\mathrm{ac}$ & 32.86 & $a b$ & 22.09 & $\mathrm{ac}$ & 20.79 & $\mathrm{ac}$ & 17.04 & $a b$ & 57.00 & $a b$ & 56.49 & a & 60.05 & a \\
\hline 13 January 2011 & 33.78 & $\mathrm{bd}$ & 33.51 & $\mathrm{ac}$ & 31.92 & $\mathrm{~b}$ & 22.76 & ac & 20.06 & ad & 16.00 & ac & 57.50 & $a b$ & 62.60 & a & 74.82 & a \\
\hline 17 February & 34.55 & $a b$ & 30.32 & de & 26.21 & ef & 21.68 & ad & 12.74 & $\mathrm{fg}$ & 6.99 & df & 58.24 & $a b$ & 82.46 & a & -34.17 & $\mathrm{bc}$ \\
\hline 10 March & 36.17 & $a$ & 34.40 & $a b$ & 25.62 & ef & 22.39 & ac & 21.28 & $a b$ & 5.23 & ef & 59.64 & $a b$ & 60.51 & a & -31.22 & bc \\
\hline 23 March & 36.01 & a & 31.92 & $\mathrm{~cd}$ & 24.35 & $f$ & 24.15 & $a b$ & 13.22 & eg & 4.25 & ef & 59.89 & $a b$ & 76.06 & a & -32.30 & bc \\
\hline 08 April & 34.70 & $a b$ & 33.60 & $\mathrm{ac}$ & 27.71 & de & 20.70 & be & 19.14 & ad & 7.88 & de & 60.99 & $a b$ & 61.80 & a & -0.96 & $\mathrm{~b}$ \\
\hline 25 April & 36.20 & a & 34.61 & $a b$ & 26.63 & ef & 25.07 & a & 21.72 & a & 5.48 & ef & 61.20 & $a b$ & 60.72 & a & -72.41 & C \\
\hline 10 May & 32.30 & $d$ & 32.91 & $\mathrm{ac}$ & 24.35 & $f$ & 17.40 & de & 17.36 & $\mathrm{bd}$ & 2.98 & $f$ & 65.31 & $a b$ & 65.28 & a & -48.48 & bc \\
\hline 24 May & 30.42 & $\mathrm{e}$ & 29.60 & e & 30.01 & bd & 12.16 & $f$ & 10.44 & $g$ & 11.30 & $\mathrm{~cd}$ & 70.93 & $a$ & 70.46 & a & 70.72 & a \\
\hline 13 June & 27.37 & $f$ & 26.59 & $f$ & 26.98 & ef & 7.16 & $g$ & 6.16 & $\mathrm{~h}$ & 6.66 & df & 26.69 & $\mathrm{~b}$ & -32.48 & $b$ & -33.05 & $\mathrm{bc}$ \\
\hline LSD & 1.8 & & 2.31 & & 3.01 & & 4.35 & & 4.03 & & 4.76 & & 40.5 & & 40.71 & & 49.83 & \\
\hline 08 October 2012 & 36.46 & ac & 34.69 & $a b$ & 31.17 & $a b$ & 23.31 & C & 21.47 & $a$ & 14.89 & $a$ & 53.64 & $a b$ & 53.34 & $a$ & 64.82 & $a$ \\
\hline 05 November & 37.89 & $a b$ & 35.82 & a & 30.82 & $\mathrm{ac}$ & 25.44 & ac & 25.24 & a & 14.50 & a & 54.68 & $a b$ & 55.81 & a & -11.15 & $\mathrm{~b}$ \\
\hline 21 November & 37.32 & $a b$ & 36.41 & a & 30.08 & ad & 24.53 & $\mathrm{bc}$ & 23.82 & a & 15.36 & a & 54.23 & $a b$ & 54.26 & a & 68.11 & a \\
\hline 12 December & 37.33 & $a b$ & 34.87 & $a b$ & 32.77 & a & 28.97 & a & 21.25 & a & 14.06 & a & 58.11 & $a b$ & 55.84 & a & 81.53 & a \\
\hline 03 January 2013 & 35.99 & $\mathrm{bc}$ & 37.08 & a & 29.96 & ad & 28.14 & $a b$ & 25.43 & a & 11.31 & $a b$ & 57.17 & $a b$ & 56.94 & a & -73.28 & $\mathrm{~b}$ \\
\hline 24 January & 34.42 & $\mathrm{C}$ & 35.91 & a & 25.92 & be & 28.73 & $a b$ & 22.67 & a & 7.94 & bc & 59.58 & $a b$ & 60.32 & a & -39.59 & $\mathrm{~b}$ \\
\hline 12 February & 37.41 & $a b$ & 34.09 & $a b$ & 25.77 & ce & 25.09 & ac & 19.02 & $a b$ & 5.11 & c & 57.45 & $a b$ & -17.18 & $a b$ & -44.95 & $\mathrm{~b}$ \\
\hline 06 March & 39.08 & a & 31.82 & $\mathrm{bc}$ & 25.71 & $\mathrm{ce}$ & 27.79 & $a b$ & 12.55 & $\mathrm{bc}$ & 5.43 & c & 58.19 & $a b$ & 4.15 & $a b$ & -33.14 & $\mathrm{~b}$ \\
\hline 28 March & 36.66 & ac & 25.62 & e & 23.30 & $\mathrm{e}$ & 22.46 & c & 5.32 & c & 4.44 & c & 66.53 & $a b$ & -36.58 & $a b$ & -65.24 & $\mathrm{~b}$ \\
\hline 17 April & 36.94 & $a b$ & 29.41 & $\mathrm{~cd}$ & 24.06 & $\mathrm{e}$ & 23.00 & C & 9.02 & c & 3.75 & c & 63.68 & $a b$ & 1.65 & $a b$ & -56.40 & b \\
\hline 14 May & 31.51 & $d$ & 29.51 & $\mathrm{~cd}$ & 24.96 & de & 12.36 & d & 7.81 & c & 5.45 & c & 74.74 & $a$ & 80.03 & a & -63.37 & $\mathrm{~b}$ \\
\hline 04 June & 28.72 & $\mathrm{e}$ & 26.95 & de & 25.30 & de & 9.42 & d & 5.49 & C & 4.63 & C & -6.53 & $\mathrm{~b}$ & -82.58 & $\mathrm{~b}$ & -65.26 & $\mathrm{~b}$ \\
\hline LSD & 2.7 & & 3.1 & & 5.3 & & 4.2 & & 7.5 & & 5.6 & & 73 & & 117 & & 66.01 & \\
\hline
\end{tabular}

Table 3. The values of fruit flesh colour $\left(\mathrm{L}^{*} \mathrm{C}^{*} h^{\circ}\right)$ during harvest and post-harvest ripening process of Hass cultivar (201011 and 2012-13 harvest period)

\begin{tabular}{|c|c|c|c|c|c|c|c|c|c|c|c|c|c|c|c|c|c|c|}
\hline \multirow{3}{*}{$\begin{array}{c}\text { Harvesting time } \\
05 \text { October } 2010\end{array}$} & \multicolumn{6}{|c|}{$\mathrm{L}\left({ }^{*}\right)$} & \multicolumn{6}{|c|}{$C\left(^{*}\right)$} & \multicolumn{6}{|c|}{$\mathrm{h}^{\circ}\left({ }^{*}\right)$} \\
\hline & \multicolumn{2}{|c|}{$0^{\text {th }}$ day } & \multicolumn{2}{|l|}{$7^{\text {th }}$ day } & \multicolumn{2}{|c|}{$14^{\text {th }}$ day } & \multicolumn{2}{|l|}{$0^{\text {th }}$ day } & \multicolumn{2}{|l|}{$7^{\text {th }}$ day } & \multicolumn{2}{|c|}{$14^{\text {th }}$ day } & \multicolumn{2}{|l|}{$0^{\text {th }}$ day } & \multicolumn{2}{|l|}{$7^{\text {th }}$ day } & \multicolumn{2}{|c|}{$14^{\text {th }}$ day } \\
\hline & 65.65 & ac & 62.86 & $\mathrm{dg}$ & 59.84 & $\mathrm{de}$ & 43.40 & $\mathrm{e}$ & 44.42 & bf & 41.58 & bd & 70.76 & ac & 69.73 & $a$ & 71.06 & $a$ \\
\hline 19 October & 61.02 & ce & 62.84 & eg & 56.50 & e & 41.49 & $f$ & 42.41 & $d f$ & 44.06 & $a b$ & 67.10 & $\mathrm{fg}$ & 69.45 & $a b$ & 70.28 & $a b$ \\
\hline 03 November & 66.38 & $a b$ & 62.05 & fh & 64.21 & $\mathrm{bc}$ & 46.38 & a & 42.21 & ef & 44.27 & $a b$ & 63.49 & I & 69.02 & ac & 70.16 & $a b$ \\
\hline 23 November & 57.00 & $\mathrm{e}$ & 60.88 & gh & 56.63 & $\mathrm{e}$ & 41.33 & $f$ & 41.86 & $f$ & 36.06 & $f$ & 65.75 & gh & 67.93 & bd & 68.43 & ac \\
\hline 12 December & 60.67 & de & 63.77 & $\mathrm{cg}$ & 63.41 & $\mathrm{c}$ & 44.73 & $\mathrm{bd}$ & 44.93 & ae & 41.19 & be & 65.76 & gh & 67.91 & bd & 67.49 & bd \\
\hline 29 December & 63.35 & ad & 63.24 & $\mathrm{dg}$ & 65.71 & ac & 45.48 & ad & 45.26 & ad & 43.51 & ac & 67.32 & $f$ & 67.41 & $\mathrm{ce}$ & 66.30 & $\mathrm{ce}$ \\
\hline 13 Januar & 61.34 & ce & 59.70 & $\mathrm{~h}$ & 64.19 & $\mathrm{bc}$ & 44.47 & de & 47.81 & a & 41.71 & bd & 65.35 & $\mathrm{~h}$ & 67.36 & $\mathrm{ce}$ & 65.90 & $\mathrm{ce}$ \\
\hline uary & 64.41 & ad & 64.22 & bf & 65.20 & $\mathrm{bc}$ & 45.17 & ad & 41.78 & $f$ & 39.84 & de & 67.86 & df & 67.29 & de & 65.62 & $\mathrm{ce}$ \\
\hline & 68.25 & a & 66.83 & $a b$ & 64.23 & $\mathrm{bc}$ & 45.98 & $a b$ & 43.82 & cf & 40.19 & ce & 68.94 & $\mathrm{ce}$ & 67.23 & de & 65.26 & ce \\
\hline & 66.78 & $a b$ & 65.79 & ad & 65.22 & $\mathrm{bc}$ & 45.77 & ac & 42.00 & ef & & de & 68.38 & df & 67.12 & de & 65.25 & $\mathrm{ce}$ \\
\hline 08 April & 63.24 & bd & 66.51 & $\mathrm{ac}$ & 62.61 & $\mathrm{~cd}$ & 44.69 & $\mathrm{~cd}$ & 44.24 & cf & 41.80 & bd & 67.47 & ef & 66.90 & de & 65.22 & ce \\
\hline pril & 65.73 & $\mathrm{ac}$ & 66.20 & $\mathrm{ac}$ & 64.61 & $\mathrm{bc}$ & 44.60 & $\mathrm{ce}$ & 44.50 & bf & 38 & ef & 70.30 & ac & 66.27 & df & 65.09 & $\mathrm{ce}$ \\
\hline & 68.26 & $a$ & 67.84 & $\mathrm{a}$ & 68.89 & $a$ & & $\mathrm{bd}$ & 44.90 & ae & 42 & bd & 69.31 & bd & 65.91 & ef & 64.93 & ce \\
\hline 24 May & 67.08 & $a b$ & 68.07 & a & 67.57 & $a b$ & 45.94 & $a b$ & 45.90 & ac & 45.92 & $a$ & 70.85 & a & 65.78 & ef & 64.32 & de \\
\hline \multirow[t]{2}{*}{13 June } & 63.44 & ad & 65.66 & ae & 64.55 & $\mathrm{bc}$ & 46.14 & $\mathrm{a}$ & 47.27 & $a b$ & 46.71 & a & 70.82 & $a b$ & 64.59 & $f$ & 63.85 & $\mathrm{e}$ \\
\hline & \multicolumn{2}{|c|}{4.94} & \multicolumn{2}{|c|}{2.94} & \multicolumn{2}{|c|}{3.39} & \multicolumn{2}{|l|}{1.25} & \multicolumn{2}{|l|}{2.96} & \multicolumn{2}{|c|}{3.33} & \multicolumn{2}{|l|}{1.52} & \multicolumn{2}{|l|}{1.69} & \multicolumn{2}{|c|}{3.56} \\
\hline 08 October 2012 & \multicolumn{2}{|c|}{$65.38 a$} & 66.65 & $a b$ & 63.67 & & 44.95 & $\mathrm{~cd}$ & 47.24 & $a$ & 49.21 & $a$ & 65.68 & $\mathrm{~h}$ & 67.28 & $a b$ & 67.88 & $a b$ \\
\hline ember & 65.92 & & 68.09 & & 62. & & 46 & $a$ & 45.74 & ac & 40 & c & 66.40 & gh & 68.22 & a & 49 & $a b$ \\
\hline $21 \mathrm{Nov}$ & 65.00 & & 66.80 & & 67.0 & & 43.75 & $\mathrm{e}$ & 45.20 & ac & 42.67 & bc & 68.91 & be & 65.58 & $a b$ & 66.93 & $a b$ \\
\hline 12 December & 67.51 & & 61.46 & & 71.64 & & 46.12 & ac & 43.50 & be & 40.92 & C & 69.00 & $\mathrm{bd}$ & 65.38 & $a b$ & 67.16 & $a b$ \\
\hline 03 Janı & 51.73 & & 68.02 & & 67.86 & & 46.17 & $a b$ & 42.82 & ce & 42.14 & bc & 67.61 & eg & 66.73 & $a b$ & 65.94 & $a b$ \\
\hline inuary & 69.61 & & 69.24 & & 69.52 & & 46.11 & ac & 43.78 & ae & 40.45 & c & 68.14 & df & 67.06 & $a b$ & 67.15 & $a b$ \\
\hline 12 February & 66.79 & & 65.74 & $a b$ & 58.75 & & 46.80 & a & 41.59 & de & 38.71 & c & 66.98 & fh & 64.82 & $b$ & 63.80 & $a b$ \\
\hline 06 March & 69.79 & & 63.33 & $a b$ & 65.84 & & 45.12 & $\mathrm{bd}$ & 43.02 & ce & 41.11 & c & 68.51 & de & 64.29 & $b$ & 66.83 & $a b$ \\
\hline 28 March & 70.52 & & 67.52 & $a b$ & 63.82 & & 44.75 & de & 40.26 & e & 39.14 & c & 68.67 & ce & 67.37 & $a b$ & 73.16 & $a b$ \\
\hline 17 April & 67.44 & & 66.58 & $a b$ & 63.37 & ac & 44.35 & de & 43.94 & ad & 39.66 & c & 70.03 & ac & 68.21 & a & 73.21 & $a b$ \\
\hline 14 May & 68.65 & & 67.52 & $a b$ & 68.44 & & 47.25 & $a$ & 46.66 & $a b$ & 47.53 & $a b$ & 70.68 & a & 68.31 & a & -1.98 & $b$ \\
\hline 04 June & 66.74 & & 63.98 & $a b$ & 61.50 & & 46.76 & $a$ & 45.47 & $\mathrm{ac}$ & 42.32 & $\mathrm{bc}$ & 70.23 & $a b$ & 68.57 & a & 77.50 & $a$ \\
\hline LSD & 12.7 & & 7.04 & & 9.16 & & 1.18 & & 3.54 & & 5.47 & & 1.38 & & 3.32 & & 77.70 & \\
\hline
\end{tabular}

$\left(^{*}\right)$ The differences between the averages indicated by the same letters in the same column were not statistically significant $(p>0.05)$. 
firmness is one of the most reliable and accepted methods for assessing the maturity and ripening of avocado (Ginsberg, 1985; Magzawa and Tesfay, 2015) and the firmness gradually change depending on the maturity or ripening process of fruit (Magzawa and Tesfay, 2015). However, due to the rapid development of decay and other internal disorders as a progressive stage of ripening after harvest, determination of the firmness value of the fruit flesh is also great importance (White and Woolf, 2007). In case it is used as a measure of the postharvest ripening stage; while the flesh firmness values are initially decreasing at the intermediate level, then the reduction rate increases and flesh firmness falls to near-zero level at the fully ripening stage of fruit (Magzawa and Tesfay, 2015). When these reports were evaluated together with the other studies that made in Mexico (Villa-Rodríguez et al., 2010; Osuna-Garcia et al., 2010), in New Zealand (Cox et al., 2004) and in Turkey (Bayram et al., 2016), the similar results were also obtained in the present study and flesh firmness values decreased.

Nevertheless, for the determination of the physiological maturity of avocado, the firmness of fruit flesh has very little (Kruger et al., 1995). Although, avocado consumers have the ability to distinguish between immature fruits and those ready to eat, in terms of firmness values, they cannot distinguish fruits from each other that are in different stages of maturity (Magzawa and Tesfay, 2015). Therefore, it was conducted a study investigating the relationship between the ripening and quality characteristics in fruits of Hass cultivar having at different maturity levels in Mexico by Osuna-Garcia et al. ( 2011). According to this study, although the firmness of fruit flesh was affected from the harvesting time, there was no certain relationship between firmness and determination of the degree of ripening, and between blackening degree of the fruit skin and reduction of firmness of fruit flesh. On the other hand, in case of the firmness value of the ripening fruit flesh in the Hass cultivar is between 4.4-6.7 $\mathrm{N}$ or less, it was reported that it increased of the consumers' purchase desire (Gamble et al., 2010; Obenland et al., 2012). In addition, the blackening of the fruit skin completely and 5-15 $\mathrm{N}$ of the firmness value of the fruit flesh were accepted as adequate for eating the fruit (Osuna-Garcia et al. 2011).

In the Hass cultivar, statistically significant differences were determined in fruit weight loss (\%) according to harvest dates, maturity and ripening of fruits. It was found that the fruit weight loss were found to be higher in early (October-November) and late (April-June) harvests. However, as the maturity level of the fruit increased, the weight loss decreased due to the postharvest ripening process ( $7^{\text {th }}$ and $14^{\text {th }}$ days). Furthermore, the fruit weight loss of the fruit also changed according to the temperature and humidity of the ripening ambient. Moreover, the taste analyses, depending on the ripening of the fruit, were made at the $7^{\text {th }}$ day and/or $14^{\text {th }}$ day, and the highest were reached between January and April. It has been reported in many studies that the weight loss (\%) of the fruit has decreased according to the harvesting time together with an increase of fruit maturity (Lee, 1981b; Vakis et al., 1985; Osuna-Garcia et al. 2011; Bayram and Tepe, 2018). In a study in Greece (Vakis et al., 1985), from the beginning of December until the first week of January, the fruit weight loss was measured once a week at $20^{\circ} \mathrm{C}$ and were generally seen to decrease. According to the study made in New Zealand by Requejo-Tapia et al. (1999), the fruits collected in November and January ripened in 14 days at $15^{\circ} \mathrm{C}$, while losses of the fruit weight were founded as $2.7 \%$ in Far North and $3.8 \%$ in Te Puke. There is an inverse relationship between maturity and ripening process of avocado (Vakis et al., 1985) and weight loss varies according to the ripening degree of fruit (Osuna-Garcia et al., 2010). Therefore, in early harvests of the fruits that cannot be ripened less than 10-11 days time (Lee, 1981a; Vakis et al., 1985), a large amount of weight loss in fruits along with wrinkling of the fruit skin was determined. It was also reported that increase of the weight loss depend on the ripening degree associated with blackening of the fruit skin (Osuna-Garcia et al., 2011). It was observed that the obtained results were similar with these reports, and that the weight loss was directly affected by maturity and ripening of fruit.

According to the taste analysis done in California, it has been reported that the palatability of Fuerte cultivar rapidly increased along with rising of maturity and oil accumulation in fruit from September to January (Lee et al., 1983). In another study conducted in California (Obenland et al., 2012); fruit samples of Hass cultivar taken from two commercial packaging houses, and fruits that were imported from Mexico, Peru and Chile, were analysed in terms of taste between April 2009 and September 2010. According to these analyses; with the increase of maturation in fruit, it was stated that more soft and smooth of fruit flesh, creamier and less watery of fruit texture, and less grassy and richer taste of eating quality occurred. As a result, the acceptability of the fruit increased together with increasing the palatability (Lee, 1981a; Mizrach et al., 1999; Obenland et al., 2012; Kassim et al., 2013). In the fruits of Hass cultivar harvested in Spain at 3 different times (December 2011, January and March 2012), and which were ripened at $22^{\circ} \mathrm{C}$ and $90 \%$ relative humidity, some external and internal quality features were examined by sensory analysis and no significant relation could be detected. However, the quality of fruits was found to be at high levels during the harvest season from December to March (Cañete et al., 2018).

The colour values of fruit skin (Lab), the brightness and green color of the fruit in both 
harvest periods usually decreased from October to June, which is observed to be at a higher level between May and June. In addition, although there was a slight increase between October-April in yellow color during both harvesting periods, a very high decrease was observed between May and June. At the end of the harvest periods, the fruits usually turned into a darker, dull and purplish-black appearance to according the beginning of the harvest. When the ripening process $\left(0^{\text {th }}, 7^{\text {th }}\right.$ and $14^{\text {th }}$ day) were examined; along with increasing fruit maturity during both harvest periods, it was observed in fruit skin that the brightness values decreased at a higher level between January and May and that the green and yellow colour values reduced at a higher level between the October and April. However, the reduction rate of the brightness, green and yellow colour in later periods was in lower levels.

Although there is no external and physical change in the fruit during maturation, the skin color of some cultivars changes from green to light green (Magzawa and Tesfay, 2015). In the fruit skin of the Hass cultivar, there is transforming from fully green colour to the different degrees of blackness (OsunaGarcia et al., 2010; Osuna-Garcia et al., 2011). As in this study, although the skin colour is one of the indicators being a help to determine of the fruit quality of avocado (Kassim et al., 2013) and is different according to the harvest dates, it is not possible to state a very fast and clear change in the colour values. Therefore, the determination of maturity according to only colour values of fruit flesh is insufficient (Magzawa and Tesfay, 2015). However, along with a delaying harvest, the fact that the fruit skin of Hass cultivar firstly turning from green to purple and after turning into the black color shows that the skin colour can be used as a sign of maturity (Cox et al., 2004; Magzawa and Tesfay, 2015). Color change on the skin during the ripening process of fruits does not cause a problem in the green-skinned cultivars (if there is no disease and spotting), while it can cause problems in blackening by ripening cultivars such as Hass (Hofman et al., 2002). Despite the fact that the blackening of fruit skin of Hass cultivar is an indication of maturity characterized by low fruit flesh firmness and short shelf life (Hofman et al., 2002; Osuna-Garcia et al., 2010; Osuna-Garcia et al., 2011), it was determined that it was not associated with fruit quality (OsunaGarcia et al., 2011). Although there were significant differences between the harvest dates in terms of fruit flesh color, it was found that fruit flesh color is not sufficient to determine maturity by itself, and similar results were obtained with Hofman et al. (2002) and Bayram and Tepe (2018).

The correlation coefficients $(r)$ for each harvest periods were calculated between harvesting time with ripening and maturity, and between ripening and maturity. These correlation coefficients $(r)$ are given in Table 4.
Throughout the ripening $\left(0^{\text {th }}, 7^{\text {th }}\right.$, and $14^{\text {th }}$ day), there was a very high positive correlation between harvesting time and dry matter. It was determined that there was a negative relationship between harvest time and fruit flesh firmness, especially in the 2012-2013 harvest period. Between harvest time and fruit skin colour, it was observed that there was usually a relationship during the ripening process $\left(0^{\text {th }}, 7^{\text {th }}\right.$, and $14^{\text {th }}$ day), especially in the 2012-2013 harvest period. In addition, during the ripening period $\left(0^{\text {th }}, 7^{\text {th }}\right.$, and $14^{\text {th }}$ day), while a positive correlation was detected between dry matter values, positive and negative correlations were detected between the fruit skin values.

At the beginning of the ones should do to increase the competitiveness of the avocado industry and to preserve of existing confidence of consumers in the purchased product, there is need to introduce fruits to the market with consistent quality with predictable ripening (Magzawa and Tesfay, 2015). Therefore, depending on the processing of the fruit to the product and the transportation distance, fruits should be harvested according to physiological and horticultural maturity level (Magzawa and Tesfay, 2015). The maturity of avocado as horticultural characteristics was defined as the period that the harvested fruit is smoothly softened and have a minimum acceptable taste (Blumenfeld et al., 1992). As for the physiological maturity of avocado found generally correlated in a high degree with the maturity determined by taste analysis, and according to the occurrence of acceptable taste in the fruit occurred close to the same periods in each year (Lee, 1981a). In addition, the time of reaching to this maturity can vary from year to year and up to 3 weeks (Blumenfeld et al., 1992). Making a decision to harvest as a commercial for the avocado producers is very difficult because the fruits do not demonstrate easily identifiable physical properties when they reach to maturity. (Lee, 1981a; Olarewaju, 2014). In many countries where avocado is grown, according to the quality characteristics of fruit detected in an ongoing process before and after the harvest, it has been tried to separately determination of the fruit maturity and harvest period for each cultivar. Therefore; the variability of many factors affecting maturity and ripening process and their relations with each other, it has been investigated with regard to the determination of the fruit quality during the harvest period and encouraging the purchasing desire of producers.

When a single index is used to determine of maturity in the fruit; although it has reached the desired values, this index should adequate and protective to determine maturity standard and prevent marketing of fruits that are not on acceptable quality in terms of ripening (Hofman et al., 2002). Although the percentage of dry weight is a relatively useful method as a maturity standard, it is recommended to continue the studies for 
Bayram and Tepe / HortiS, 37(2):102-112

Table 4. The correlation coefficients $(r)$ calculated for each harvest periods

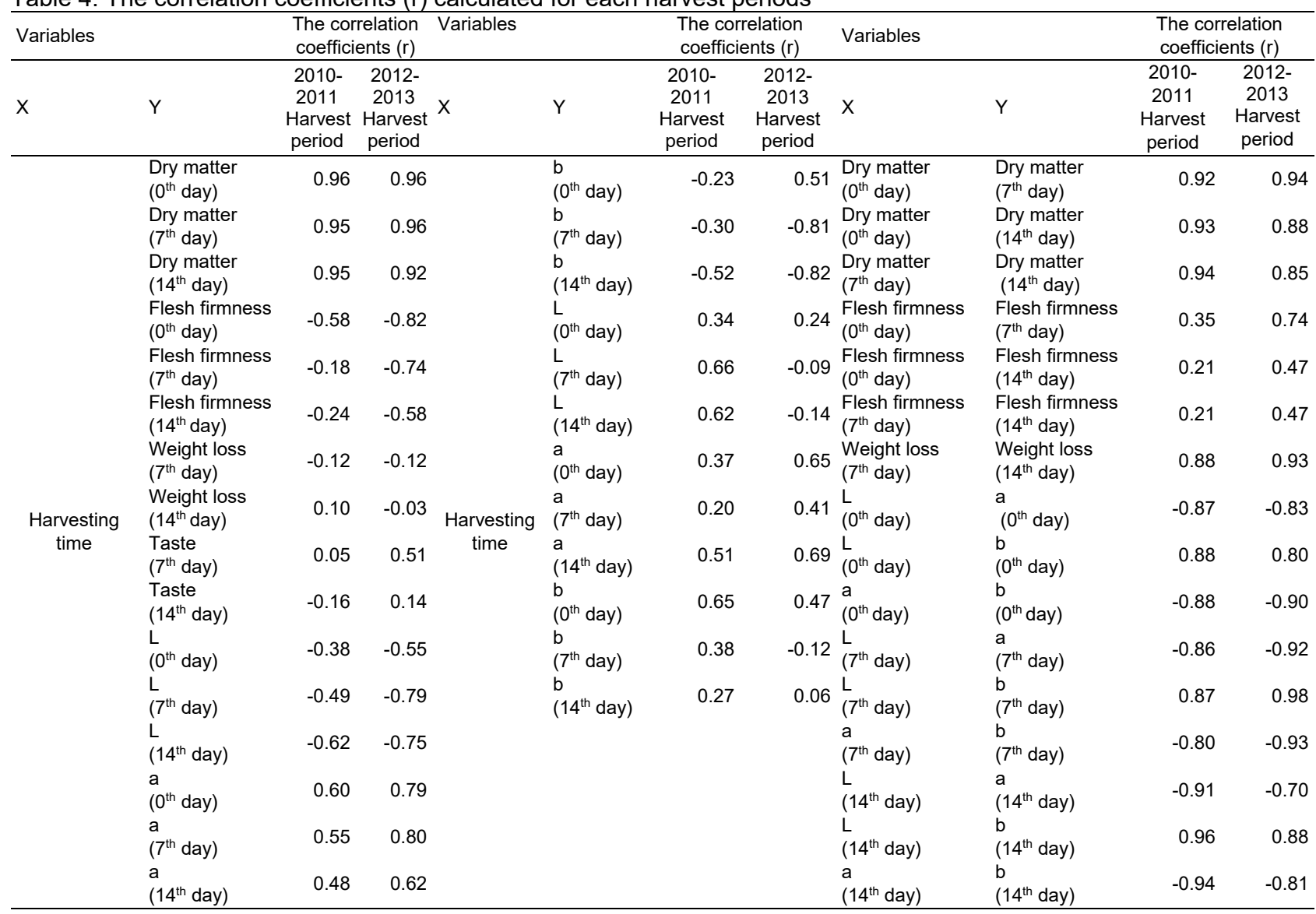

developing another standard due to finding sometimes its poor relationship with mature fruit quality (Hofman et al., 2002). Measurements determining of the fruit quality such as fruit skin colour, fruit flesh firmness and dry weight ratio, in some cases unable to predict the variable ripening of fruit in post-harvest (Hernández et al., 2015; Rivera et al., 2017). Climate, soil and agricultural management conditions can have an effect on maturation variability (Coggins, 1984; Rivera et al., 2017). In the Hass cultivar, observed ripening variability or heterogeneity of ripening is the result of complex fruit physiology associated with preharvest and post-harvest factors (Hernández et al., 2015). Therefore, attempting to predict post-harvest behaviours by considering only a single pre-harvest variable may be a deceptive simplification of reality (Rivera et al., 2017) and if two maturity standards such as DM\% and healthy ripening capacity are used together, the marketing risk of immature fruit reduces (Hofman et al., 2002).

In a study conducted by Pak et al. (2003) in New Zealand, the significant relationships have been observed between dry matter content and some fruit quality characteristics (especially in export control). According to this study, as the process of maturation of the fruit progressed, the rate of vascular fibrousness and decay in fruit decreased. It was found that the minimum dry matter content for fruit maturity was an acceptable index and increased in a linear line between July and September. However, the rates of dry matter accumulation in the fruit vary considerably at a certain harvest time (at the beginning and end of the season) and between harvest times. In general, when the dry matter rate is above $24.0 \%$, it is the recommended ratio for early harvest as whole fruit flesh is more smoothly ripening and have better quality features in the post-harvest process. In another study made with Hass cultivar in New Zealand (Gamble et al., 2010); along with the increase in dry matter (between 22.0-27.0\%), it has been reported that consumers' desire to buy increased. In the study in Colombia (Calvalho et al., 2014), as an acceptable level by the consumers, it was reported that dry matter rate needs to be between $22.0-26.0 \%$ with at least $11.2 \%$ minimum oil content in Hass cultivar. In a study conducted in Michoacán where $80.0 \%$ of avocado orchards were found in Mexico (Osuna-Garcia et al., 2011); although the harvest time of Hass cultivar is between September and April, it is stated that the dry matter content was between $21.5-28.0 \%$ in a certain period (mid-October and early January), which the ripening of the fruit is regular and the shelf life is at good level. For acceptable taste values for Hass cultivar, it was reported that it was reached in early December in California (Lee et al., 1983) and 
in the second week of December in Greece (Vakis et al., 1985). In a study conducted for determination with various aspects of product quality of Hass cultivar and at the same time quantitative analysis of consumer preferences (Gamble et al., 2010); it was evaluated that dry matter rates ranged between $20.0 \%$ (minimum mature) and $40.0 \%$ (very mature) levels and found in the different stages of flesh firmness. As the rate of dry matter in the fruit increases, there has been a constant increase in the willingness and intention of consumers to buy. According to the results of this study, the consumers' preference maturity level for avocado was determined as values that the firmness of fruit flesh was $6.5 \mathrm{~N}$ or less and the dry matter content was between $22.0-27.0 \%$. In another study, it was reported that dry weight rate for avocado distributed between the content values greater than $35.0 \%$, which the fruit was most suitably process, and fewer values than $20.0 \%$, which the taste and quality of fruit too low to sell (Clark et al., 2007).

In the research conducted with the Hass variety in Mexico; while the fruit skin colour firstly transformed from green to purple colour with the increase in harvest maturity, the fruit skin colour became blacking in the following stages along with the increase in avocado maturity index values (VillaRodríguez et al., 2010; Osuna-Garcia et al., 2011). Although this colour change in the fruit skin is accepted to be a very important index of maturity for both consumers and producers in avocado industry (Cox et al., 2004), these fruits are undesirable in some countries because it is thought to be associated with low fruit flesh firmness and short shelf life (Osuna -Garcia et al., 2010; Osuna-Garcia et al., 2011). Therefore, in the studies aimed at revealing the relationship between skin color and fruit quality, it was reported that the blackening of the fruit skin could not be associated with low fruit quality. However, it was stated that these fruits had lower firmness of fruit flesh according to the analysis made during the packaging process (Osuna-Garcia et al., 2010; Osuna-Garcia et al., 2011).

Fruits of Hass cultivar exported from Mexico to Canada was investigated during the harvest season (between October 2007 and April 2008) as shelf life and fruit quality (Osuna-Garcia et al., 2010). As a result, the dry matter content of the fruit flesh increased with the harvesting time and the degree of blackening of the fruit skin. Although the weight loss in fruit decreased with harvest date, it increased along with the degree of blackening of the skin. At the same time, even though the harvesting time had a significant effect on the firmness of the fruit flesh, there was no correlation between the blackening degree of the fruit skin and the decrease of firmness of fruit flesh. In similar with these reports, due to the occurrence of some problems in the ripening of the early or late-harvested fruit of Hass cultivar, the harvest time has been divided into 3 different periods by considering the physical and chemical development of the fruits (Bayram and Tepe, 2019). Each of the harvest periods has been defined according to the maturity and ripening of the fruit.

\section{Conclusion}

In this study, depending on the maturity and ripening of the fruit, the most suitable and acceptable harvest period for the Hass cultivar was generally determined. For the determination of the harvest maturity, observation of changes on fruit flesh firmness and in fruit skin colour takes a long time, moreover, taste analyses and fruit weight loss takes between 7-14 days, thus, these prevent to make a fast decision for marketing. During the maturation period, a very high positive correlation $(r=0.92-0.96)$ was observed between harvest time and dry matter. Furthermore, while the positive correlations at high-level $(r=0.85-0.94)$ between the dry matter values were determined, the positive and negative correlations at high-levels between colour values of the fruit skin were found.

As a result, it was found that the most reliable maturity index was dry weight content and that it had a direct relationship with harvesting time. In cases where these index values were insufficient, the other postharvest analyses (taste, fruit skin colour, and fruit flesh firmness and fruit weight loss) were helpful for the determination of the maturity of Hass cultivar. The harvesting time of Hass cultivar was determined for the three different periods divided as early, optimum (most suitable) and late harvest. $23-25 \%$ dry weight content between midOctober and late December as early harvest, 26$37 \%$ dry weight content between early of January and end of May as optimum harvest, and $38 \%$ dry weight content between beginning and end of June was determined as the late harvest.

\section{References}

Anonymous, (2005). The World Avocado Market. In; Loillet, D., \& Impert, E. (Eds)., FruiTrop Focus. CiradFlhor TA 50/PS4 34398 Montpellier Cedex, France.

Anonymous, (2006). Avokado. Akdeniz İhracatçılar Birliği. Pp: 27, (in Turkish).

Arpaia, M. L., Boreham, D., Collin, S., Fjeld, K., \& Sievert, J. (2003). Hass matury project. In: Witney, G. (Ed.) Proceedings of the California Avocado Research Symposium, pp. 120-124.

Bayram, S., \& Aşkın, M.A. (2006). Using of oil and dry matter parameters in some avocado cultivars for determination of harvest date. Süleyman Demirel University Journal of Agriculture Faculty, 1(2):38-48.

Bayram, S., Tepe, S., \& Toker, R. (2016). Determination of some physical and chemical changes in fruits of hass avocado cultivar during harvesting time. Derim, 33(1):14-26.

Bayram, S., \& Tepe, S. (2018). Determination of some physical and chemical changes of fruits of Edranol, Ettinger and Wurtz avocado varieties during harvest periods. Derim, 35(2):96-110. 
Bayram, S., \& Tepe, S. (2019). Determination of some physicochemical properties in fruits of some avocado (Persea americana Mill.) cultivars during the harvesting periods. Derim, 36(1):1-12.

Blumenfeld, A., Offer, R., Elimelech, M., Degani, C., \& El Bazri, R. (1992). Avocado fruit maturation and criteria for harvest. Proceeding of Second World Avocado Congress, pp: 489.

Cañete, M.L., Pérez de los Cobos, R., \& Hueso, J.J. (2018). Influence of harvest date on sensory quality attributes of avocado 'Hass'. Acta Horticulturea, 1194:1127-1131.

Carvalho, C.P., Velásquez, M.A., \& Rooyen, Z.V. (2014). Determination of the minimum dry matter index for the optimum harvest of 'Hass' avocado fruits in Colombia. Agronomía Colombiana, 32(3):399-406.

Clark, C.J., White, A., Jordan, R.B., \& Woolf, A.B. (2007). Challenges associated with segregation of avocados of differing maturity using density sorting at harvest. Postharvest Biology and Technology, 46(2):119-127.

Coggins, Jr. C.W. (1984). Feasibility of using fruit size and percentage dry weight to predict maturity. California Avocado Society Yearbook, 68:145-160.

Cox, K.A., McGhie, T.K., White, A., \& Woolf, A.B. (2004). Skin colour and pigment changes during ripening of 'Hass' avocado fruits. Postharvest Biology and Technology, 31:287-294.

Degani, C., Bechor, V., Albazri, R., \& Blumenfeld, A. (1986). Dry weight content as an index for determination of maturity of avocado fruits. Alon Hanotea, 40:1017-1022.

FAO. (2019). FAOSTAT Database. http://www.fao.org/faostat/en/\#data/QC. Access date: May 25, 2019.

Flitsanov, U. Mizrach, A., Liberzon, A., Akerman, M., \& Zauberman, G. (2000). Measurement of avocado softening at various temperatures using ultrasound. Postharvest Biology and Technology, 20:279-286.

Gamble, J, Harker, F.R., Jaeger, S.R., White, A., Bava, C., Beresford, M., Stubbings, B., Wohlers, M., Hofman, P.J., Marques, R., \& Woolf, A. (2010). The impact of dry matter, ripeness and internal defects on consumer perceptions of avocado quality and intentions to purchase. Postharvest Biology and Technology, 57:35-43.

Ginsberg, L. (1985). Post harvest physiological problems of avocados. South African Avocado Growers' Association Yearbook, 8:8-11.

Gonzales, A.T., Mazariegos, R.M., \& Cantwell, M. (1992). Inactivation in situ of polyphenol oxidase in ripe avocado fruit. Proceedings of Second Avocado Congress, 21-24 April, University of California, Riverside, California, pp: 409-416.

Hernández, I., Fuentealba, C., Olaeta, J.A., Lurie, S., Defilippi, B.G., Campos-Vargas R., \& Pedreschi, R. (2015). Factors associated with postharvest ripening heterogeneity of 'Hass' avocados (Persea americana Mill). Fruits, 71(5):259-268.

Hofman, P.J., Jobin-Décor, M., \& Giles, J. (2000). Percentage of dry matter and oil content are not reliable indicators of fruit maturity or quality in lateharvested 'Hass' avocado. HortScience, 35(4):694695.

Hofman, P.J., Fuchs, Y., \& Milne, D.L. (2002). Harvesting, Packing, Postharvest Tecnology, Transport and Processing. 14, pp. 363-390. In: Whiley, A.W., Schaffer, B. and Wolstenholme, B.N. (Eds), The
Avocado: Botany, Production and Uses; Cabi Publishing.

Kassim, A., Workneh, T.S., \& Bezuidenhout, C.N. (2013). A review on postharvest handling of avocado fruit. Academic Journal, 8(2):2385-2402.

Knight, Jr. R.J. (2002). History, Distribution and Uses. In: A.W. Whiley, B.Schaffer And B.N. Wolstenholme (Eds) The Avocado: Botany, Production and Uses; Cabi Publishing, 1:10.

Kruger, F.J., Stassen, P.J.C., \& Snijders, B. (1995). A preliminary study on variation in the maturity parameters of avocados from the kiepersol/hazyview area. South African Avocado Growers' Association Yearbook, 18:67-73.

Lee, S.K. (1981a). Maturity Studies of Avocado (Persea amaricana Mill.) Fruit in California. PhD Thesis University of California Riverside, USA.

Lee, S.K. (1981b). A review and background of the avocado maturity standard. California Avocado Society Yearbook, 65:101-109.

Lee, S.K., \& Coggins, Jr.C.W. (1982). Dry weight method for determination of avocado fruit maturity. California Avocado Society Yearbook, 66:67-70.

Lee, S.K., Young, R.E., Schiffman, P.M., \& Coggins, C. W.Jr. (1983). Maturity studies of avocado fruit based on picking dates and dry weight. Journal of the American Society for Horticultural Science, 108(3):390-394.

McGuire, R.G. (1992). Reporting of objective color measurements. HortScience, 27:1254-1255.

Magzawa, L.S., \& Tesfay, S.Z. (2015). A review of destructive and non-destructive methods for determining avocado fruit maturity. Food and Bioprocess Technology, 8(10):1995-2011.

Mizrach, A., Flitsanov, U., El-Batsri, R., \& Degani C. (1999). Determination of avocado maturity by ultrasonic attenuation measurements. Scientia Horticulturae, 80:173-180.

Naamani, G. (2007). Developments in the avocado world. California Avocado Society Yearbook, 90:71-76.

Newett, S.D.E., Crane, J.H., \& Balerdi, C.F. 2002. Cultivars and Rootstocks. In: A.W.Whiley, B. Schaffer and B.N. Wolstenholme, The Avocado: Botany, Production and Uses, Pp: 162-169. Cabi Publishing.

Obenland, D., Collin, S., Sievert, J., Negm, F., \& Arpaia M.L. (2012). Influence of maturity and ripening on aroma volatiles and flavor in 'Hass' avocado. Postharvest Biology and Technology, 71:41-50.

Olarewaju, O.O. (2014). Evaluation of Maturity Parameters of 'Fuerte' and 'Hass' Avocado Fruit. Discipline of Horticultural Science, School of Agricultural, Earth and Environmental Sciences, College of Agriculture, Engineering and Sciences University of KwaZulu-Natal Pietermaritzburg, South Africa. The Thesis of Master, Pp: 139.

Osuna-García, J.A., Doyon, G., Salazar-García, S., Goenaga, R., \& González-Durán I.J.L. (2010). Effect of harvest date and ripening degree on quality and shelf life of hass avocado in Mexico. Fruits, 65:367375.

Osuna-García, J.A., Doyon, G., Salazar-García, S., Goenaga, R., \& González-Durán I.J.L. (2011). Relationship between skin color and some fruit quality characteristics of 'Hass' avocado. Journal of Agriculture of the University of Puerto Rico, 95(12):15-23. 
Ozdemir, F., Topuz, A., Demirkol, A., \& Gölükcü, M (2003). Changes in composition of some avocado (Persea americana Mill.) cultivars during harvesting time and postharvest ripening period. Gida, 29(2):177-183.

Ozdemir, F., \& Topuz, A. (2004). Changes in dry matter oil content and fatty acid composition of avocado during harvesting time and post-harvesting ripening period. Food Chemistry, 86:79-83.

Pak, H.A., Dixon, J., \& Cutting, J.G.M. (2003). Influence of early season maturity on fruit quality in New Zealand Hass avocados. Proceedings V World Avocado Congress, 19-24 October, Malaga, Spain, pp: 635-640.

Parodi, G., Sanchez, M., \& Daga, W. (2007). Correlation of Oil Content, Dry Matter and Pulp Moisture As Harvest Indicators in Hass Avocado Fruits (Persea americana Mill) Grown under Two Conditions of Orchards in Chincha-Perú. Proceedings VI World Avocado Congress (Actas VI Congreso Mundial del Aguacate), Viña Del Mar, 12-16 November, Chile.

Requejo-Tapia, L.C. Woolf, A.B. Roughan, G. Schroeder, R. Young, H., \& White, A. (1999). Avocado postharvest research: 1998/99: seasonal changes in lipid content and fatty acid composition of 'Hass' avocados. pp. 1-25. HortResearch Client Report No. 2000/1 Contract No.5262, Report to the NZ Avocado Industry Council, New Zeland.

Rivera, S.A., Ferreyra, R., Robledo, P., Selles, G., Arpaiac, M.L., Saavedra, J., \& Defilippi, B.G. (2017) Identification of preharvest factors determining postharvest ripening behaviors in 'Hass' avocado under long term storage. Scientia Horticulturae, 216:29-37.

Wedding, B.B., Wright, C., Grauf, S., \& White, R.D. (2011). The Application of Near Infrared Spectroscopy for the Assessment of Avocado Quality Attributes, The State of Queensland, Department of Employment, Economic Development and Innovation, 13:211.
Whiley, A.W., Saranah, J.B., \& Rasmussen, T.S. (1992). Effect of time of harvest on fruit size, yield and trunk starch concentration of 'Fuerte' avocados. Proceedings of Second World Avocado Congress, 2124 April, California, pp: 155-159.

Whiley, A.W., Rasmussen, T.S., Saranah, J.B., \& Wolstenholme, B.N. (1996). Delayed harvest effects on yield, fruit size and starch cycling in avocado (Persea americana Mill.) in subtropical environments. II. The late-maturing cv. Hass. Scientia Horticuhurae, 66:35-49.

White, A., \& Woolf, A. (2007). Fruit Ripening. In: M.L. Arpaia and R. Hofshi (eds.), Proceedings of Avocado Brainstorming. Session 8. Postharvest Handling and Quality Control, October 27-28, 159-160.

Winer, L., Zachs, I., \& Chudzik, P. (2007). Avocado Fruit Growth During Winter The Importance of Irrigation Between Rains. Proceedings VI World Avocado Congress, 12 - 16 Nov. 2007, Viña Del Mar, Chile.

Woolf, A., Clark, C., Terander, E., Phetsomphou, V., Hofshi, R., Arpaia, M.L. (2003). Measuring avocado maturity; ongoing developments. The Orchardist, 4045.

Vakis, N.J., Gregoriou, C., \& Papademetriou, M. (1985). Maturity and picking dates of avocados under Cyprus conditions. California Avocado Society Yearbook, 69:81-88.

Villa-Rodríguez, J.A., Molina-Corral, F.J., Ayala-Zavala, J.F., Olivas, G.I., \& González-Aguilar, G.A. (2010). Effect of maturity stage on the content of fatty acids and antioxidant activity of 'Hass' avocado. Food Research International, 44:1231-1237.

Young, R. E., \& Lee, S.K. (1978). Avocado fruit maturity. California Avocado Society Yearbook, 62:51-57.

Zerbini, E., \& Polesello, A. (1984). Measuring the color of apple skin by different techniques. Proceedings of the Workshop on Pome-fruit Quality, pp:161-171.

Zentmyer, G.A. (1987). Avocados around the world. Avocado Society Yearbook, 71:63-77. 